\title{
Human Bêta Defensin-1 Gene (-20G/A) polymorphism in Vitiligo Egyptian Patients Sample
}

\author{
A.A.Saleh ${ }^{1}$, R.M. Salem ${ }^{1}$, A.M.AbdelRahman ${ }^{2}$ and M.G.Al-Khafaji ${ }^{1}$ \\ ${ }^{1}$ Dermatology, Venereology and Andrology Dept., Faculty of Medicine, Benha Univ., Benha, Egypt \\ ${ }^{2}$ Clinical and Chemical Pathology Dept., Faculty of Medicine, Benha Univ., Benha, Egypt \\ E-Mail: mohammedalkhafaji888@gmail.com
}

\begin{abstract}
Vitiligo is a serious cosmetic condition, mainly for individuals with dark skin. The patches of skin affected become white and usually have sharp margins. Often the patches begin on areas of skin that are exposed to the sun. It is more noticeable in people with dark skin. Vitiligo may result in psychological stress and those affected may be stigmatized. The exact cause of vitiligo is unknown. It is believed to be due to genetic susceptibility that is triggered by an environmental factor such that an autoimmune disease occurs. $\beta$-defensins are small antibiotic peptides that have direct antimicrobial, immune-enhancing and modulating and cytokine-like activity in the inflammatory signaling pathways. As a part of the innate immune system they mediate the first line of host defense and are encoded by the $\beta$-defensin genes localized in a human genome. HBD was studied as potential modulator of several autoimmune diseases. In this study, it was aimed to evaluate the association between human Beta-Defensin-1(-20 G/A) gene polymorphism with vitiligo. The study included 50 patients suffering from vitiligo and 50 healthy control subjects of matched age, sex, BMI of Egyptian origin. HBD (-20G/A) AA genotype and A allele showed significantly lower frequency in vitiligo patients when compared to the control subjects. AA genotype was significantly associated with lower VASI score.
\end{abstract}

\section{Introduction}

Vitiligo is an acquired pigmentation disorder characterized by the loss of epidermal melanocytes and/or their function. It is a relatively common disorder, with a prevalence rate of $0.2-1.8 \%$ [1].

Although the exact aetiology of vitiligo remains elusive, autoimmunity is believed to play an important role in disease pathogenesis, as vitiligo is often associated with autoimmune diseases [2].

owledged to play an important role in vitiligo pathogenesis [3].

Th17 response is characterized by the elicitation of antimicrobial peptides (AMPs) through interleukin (IL)-17A, IL17F and IL-22 signalling, leading to localized inflammation. An excess of constitutively expressed AMPs such as human beta-defensin (HBD)1 may also contribute to local inflammation; for HBD1 , this is due to its ability to chemoattract neutrophils, immature dendritic cells and $\mathrm{T}$ cells directly through chemokine receptor (CCR)6 signalling and indirectly by HBD-3 induction [4].

Moreover, defensins have an impact on antigen presentation by influencing processes of differentiation. Furthermore, defensins were demonstrated to inhibit the classic and lectin pathway of complement activation by binding $\mathrm{Clq}$ and mannose-binding lectin, they increase proliferation, migration, and proinflammatory cytokine secretion in keratinocytes, and enhance antigen-presentation and proinflammatory cytokine secretion in lung epithelial cells [5].

Certain polymorphisms in DEFB1, the gene encoding for HBD-1, may affect its transcription rate and the expression of HBD-1 protein [6]. Considering autoimmunity, HBD-1 and its gene polymorphisms have been evaluated in psoriasis (PSO), type 1 diabetes (T1D), oral lichen planus (OLP), inflammatory bowel disease (IBD), and systemic and cutaneous lupus erythematosus (SLE and CLE, respectively), with variable degrees of association identified [7]. However, the association between this gene polymorphisms and vitiligo has not been studied enough in different populations. In this study was aimed to evaluate the association between human BetaDefensin-1 (-20 G/A) gene polymorphisms and vitiligo development in a sample of Egyptian vitiligo patients.

\section{Subjects and methods}

This prospective case-control study included 100 participants. 50 patients suffering from active non segmental vitiligo, in addition to 50 apparently healthy control subjects of matched age and sex. All patients were selected from the outpatient clinic of Dermatology, Venereology and Andrology Department of Benha University Hospitals, Benha, Egypt.

Participants gave their informed written consent before enrollment and the study was approved by the Research Ethics Committee in Faculty of Medicine, Benha University.

All patients included in the study have vitiligo. Patients with skin disease other than vitiligo and subjects with acute or chronic infections, malignancies, autoimmune disorders, hepatic or renal diseases were excluded.

\section{Vasi score}

The Vitiligo Area Scoring Index (VASI) is a validated quantitative scale developed by . [8] It was initially developed to measure the response of vitiligo to narrowband ultraviolet-B treatment but has since been used to evaluate various vitiligo therapies. In this assessment, the patient's body is separated into five regions: the hands, upper extremities (including axilliary regions), trunk, lower extremities (including inguinal regions and buttocks), and the feet. 
Subsequent studies have added a sixth site: the head/neck area. The percentage of vitiligo involvement for each body region is calculated by using the palmar method. The palmar method uses the palmar surface area of the patient's hand as an estimation guide and defines the surface of the patient's hand including fingers to be $1.0 \%$ of the total body surface area. Each site is then clinically evaluated by visual assessment for the pattern of skin depigmentation using a visual scale.

Pattern of depigmentation are illustrated with a descriptive atlas of patient photographs in the article, the extent of residual depigmentation is expressed by the following percentages: $0,10 \%, 5 \%, 50 \%, 75 \%$, $90 \%$, or $100 \%$. At $100 \%$ depigmentation, no pigment is present; at $90 \%$, specks of pigment are present; at $75 \%$, the depigmented area exceeds the pigmented area; at $50 \%$, the depigmented and pigmented areas are equal; at $25 \%$, the pigmented area exceeds the depigmented area; at $10 \%$, only specks of depigmentation are present. The VASI is then derived by multiplying the values assessed for the vitiligo involvement by the percentage of affected skin for each body site and summing the values of all body sites together.

$\mathrm{VASI}=\Sigma[$ HAND UNITS $] \times[$ RESIDUAL DEPIGMENTATION]

Table (1) Demographic data of the studied groups.

\begin{tabular}{llccccc}
\hline & & \multicolumn{2}{c}{ Control } & \multicolumn{3}{c}{ Cases } \\
N=50 & & p \\
\hline Age (years) & mean \pm SD & 43.6 & 8.8 & 40.2 & 11.7 & 0.104 \\
Males & $\mathrm{N}(\%)$ & 18 & $(36)$ & 17 & $34 \%$ & 0.834 \\
Females & $\mathrm{N}(\%)$ & 32 & $(64)$ & 33 & $66 \%$ & \\
\hline
\end{tabular}

$\mathrm{SD}$, standard deviation; $\mathrm{N}$, number; $\mathrm{p}<0.05$ is considered significant.

3.2 Clinical features in all studied cases

Table (2) Clinical features in all studied cases.

\begin{tabular}{lcccc}
\hline & & & \multicolumn{2}{c}{ Cases } \\
N=50 \\
\hline Clinical types of vitiligo & & $\mathrm{N}(\%)$ & 18 & $36 \%$ \\
& Acrofacial & $\mathrm{N}(\%)$ & 2 & $4 \%$ \\
& Universal & $\mathrm{N}(\%)$ & 36 & $72 \%$ \\
Leukotrichia & Focal & $\mathrm{N}(\%)$ & 12 & $24 \%$ \\
VASI & Vulgaris & $\mathrm{N}(\%)$ & 6 & $12 \%$ \\
\hline
\end{tabular}

3.3 Both control and patients' groups were in Hardy Weinberg equilibrium regarding both SNPs Table (3).

Table (3) Hardy Weinberg equilibrium in all studied groups.

\begin{tabular}{|c|c|c|c|c|c|}
\hline & & \multicolumn{2}{|c|}{ Control $\mathbf{N}=\mathbf{5 0}$} & \multicolumn{2}{|c|}{ Cases $\mathrm{N}=50$} \\
\hline & & \multicolumn{2}{|c|}{$\begin{array}{c}\text { Distribution } \\
\end{array}$} & \multicolumn{2}{|c|}{$\begin{array}{c}\text { Distribution } \\
\end{array}$} \\
\hline & & Observed & Expected & Observed & Expected \\
\hline \multirow[t]{4}{*}{$-20 \mathrm{G} / \mathrm{A}$} & GG & 14 & 12.5 & 24 & 23.1 \\
\hline & GA & 22 & 25 & 20 & 21.8 \\
\hline & $\mathbf{A A}$ & 14 & 12.5 & 6 & 5.1 \\
\hline & $\mathbf{p}$ & \multicolumn{2}{|c|}{0.396} & \multicolumn{2}{|c|}{0.567} \\
\hline
\end{tabular}

3.4 DEFB1 -20G/A genotypes and alleles in the studied groups. 
Table (4) DEFB1 -20G/A genotypes and alleles in the studied groups.

AA genotype has a protective role against vitiligo development.

\begin{tabular}{|c|c|c|c|c|c|c|c|c|c|}
\hline & & \multicolumn{2}{|c|}{$\begin{array}{c}\text { Control } \\
\mathbf{N}=50\end{array}$} & \multicolumn{2}{|c|}{$\begin{array}{l}\text { Cases } \\
\mathbf{N}=\mathbf{5 0} \\
\end{array}$} & \multirow[t]{2}{*}{$\mathbf{p}$} & \multirow[t]{2}{*}{ OR } & \multirow{2}{*}{\multicolumn{2}{|c|}{$95 \% \mathrm{CI}$}} \\
\hline & & $\mathbf{N}$ & $\%$ & $\mathbf{N}$ & $\%$ & & & & \\
\hline \multirow[t]{6}{*}{$-20 G / A$} & GG & 14 & 28 & 24 & 48 & - & 1 & \multicolumn{2}{|c|}{ (reference) } \\
\hline & GA & 22 & 44 & 20 & 40 & 0.163 & 0.673 & 0.386 & 1.174 \\
\hline & $\mathrm{AA}$ & 14 & 28 & 6 & 12 & $0.017 *$ & 0.423 & 0.209 & 0.857 \\
\hline & $\mathrm{GA}+\mathrm{AA}$ & 36 & 72 & 26 & 52 & $0.040 *$ & 0.583 & 0.349 & 0.975 \\
\hline & $\mathrm{G}$ & 50 & 50 & 68 & 68 & - & 1 & \multicolumn{2}{|c|}{ (reference) } \\
\hline & A & 50 & 50 & 32 & 32 & 0.010 & 0.624 & 0.437 & 0.892 \\
\hline
\end{tabular}

OR, odds ratio; CI, confidence interval. Logistic regression test was used.

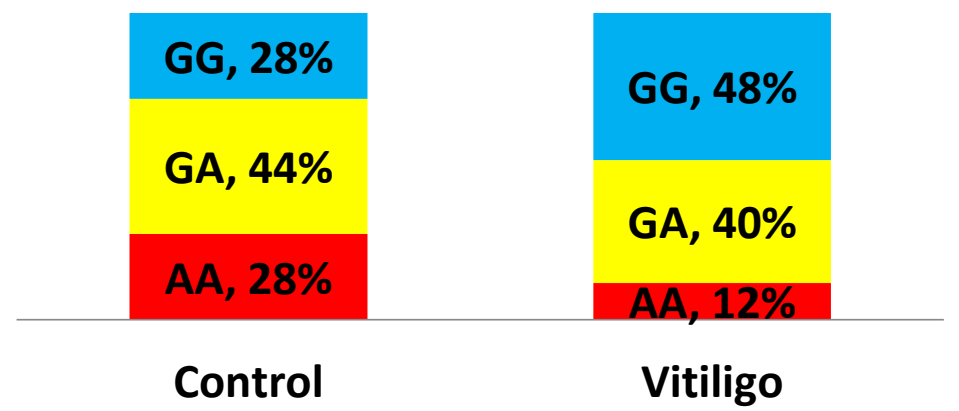

Fig (1) DEFB1 -20G/A genotypes in cases and control groups.

\subsection{HBD1 (-20G/A) SNP and the studied variables}

There was insignificant difference in HBD1 (-20G/A) genotypes distribution in relation to different demographic, history and clinical findings, excepting for the mean VASI score. AA genotype was associated with significantly lower VASI scores Table (5).

Table (5) Relation between HBD1 (-20G/A) genotypes and the studied variables.

\begin{tabular}{|c|c|c|c|c|c|c|c|c|}
\hline & & & $\begin{array}{c}\mathbf{G G} \\
\mathrm{N}=24\end{array}$ & $\begin{array}{c}\text { GA } \\
\mathbf{N}=\mathbf{2 0}\end{array}$ & $\begin{array}{c}\mathrm{AA} \\
\mathrm{N}=6\end{array}$ & $\begin{array}{c}\text { GA+AA } \\
\mathbf{N}=26\end{array}$ & $\mathbf{P}^{1}$ & $\mathbf{P}^{2}$ \\
\hline \multirow{2}{*}{\multicolumn{2}{|c|}{ Age (years) }} & mean & 43.3 & 36.2 & 40.7 & 37.2 & 0.132 & 0.066 \\
\hline & & $\pm \mathrm{SD}$ & \pm 13.4 & \pm 9.1 & \pm 9.6 & \pm 9.2 & & \\
\hline \multicolumn{2}{|l|}{ Males } & $\mathrm{N} \%$ & $833.3 \%$ & $840.0 \%$ & $116.7 \%$ & $934.6 \%$ & 0.645 & 0.924 \\
\hline \multicolumn{2}{|l|}{ Females } & $\mathrm{N} \%$ & $1666.7 \%$ & $1260.0 \%$ & $583.3 \%$ & $1765.4 \%$ & & \\
\hline \multicolumn{2}{|l|}{ Family History } & $\mathrm{N} \%$ & $625.0 \%$ & $210.0 \%$ & $233.3 \%$ & $415.4 \%$ & 0.212 & 0.490 \\
\hline \multicolumn{2}{|l|}{ BMI (Kg/m2) } & Mean \pm SD & $28.0 \pm 4.7$ & $27.3 \pm 4.6$ & $30.4 \pm 2.5$ & $28.0 \pm 4.4$ & 0.321 & 0.998 \\
\hline \multirow{4}{*}{$\begin{array}{l}\text { Provo-cating } \\
\text { factors }\end{array}$} & Stress & $\mathrm{N} \%$ & $1979.2 \%$ & $1995 \%$ & $6100 \%$ & $2596.2 \%$ & 0.628 & 0.312 \\
\hline & UVA & $\mathrm{N} \%$ & $28.3 \%$ & $00 \%$ & $00 \%$ & $00 \%$ & 0.487 & 0.465 \\
\hline & Kobneriz-ation & $\mathrm{N} \%$ & $312.5 \%$ & $15 \%$ & $00 \%$ & $13.8 \%$ & 0.476 & 0.798 \\
\hline & & & $\mathrm{GG} \mathrm{N}=24$ & $\mathrm{GAN}=20$ & $\mathrm{AAN}=6$ & $\mathrm{GA}+\mathrm{AAN}=26$ & $\mathrm{P}^{1}$ & $\mathrm{P}^{2}$ \\
\hline \multicolumn{2}{|c|}{ Age of onset (years) } & Mean \pm SD & $21.3 \pm 6.7$ & $18.4 \pm 5.9$ & $17.0 \pm 5.4$ & $18.1 \pm 5.9$ & 0.772 & 0.484 \\
\hline \multicolumn{2}{|c|}{ Duration (years) } & Mean \pm SD & $20.9 \pm 6.7$ & $17.8 \pm 5.3$ & $27.3 \pm 8.9$ & $20.0 \pm 6.3$ & 0.387 & 0.830 \\
\hline \multirow[t]{3}{*}{ Course } & Stable & $\mathrm{N} \%$ & $520.8 \%$ & $210 \%$ & $350.0 \%$ & $519.2 \%$ & 0.139 & 0.887 \\
\hline & Progressive & $\mathbf{N \%}$ & 19 & 18 & 3 & 21 & & \\
\hline & & & $79.2 \%$ & $90 \%$ & $50.0 \%$ & $80.8 \%$ & & \\
\hline \multirow{4}{*}{$\begin{array}{l}\text { Clinical types of } \\
\text { vitiligo }\end{array}$} & Acrofacial & $\mathrm{N} \%$ & $833.3 \%$ & $840 \%$ & $233.3 \%$ & $1038.5 \%$ & 0.916 & 0.706 \\
\hline & Universal & $\mathrm{N} \%$ & $28.3 \%$ & $00 \%$ & $00 \%$ & $00 \%$ & 0.606 & 0.225 \\
\hline & Focal & $\mathrm{N} \%$ & $1458.3 \%$ & $1680.0 \%$ & $6100.0 \%$ & $2284.6 \%$ & 0.083 & 0.059 \\
\hline & Vulgaris & $\mathrm{N} \%$ & $833.3 \%$ & $420.0 \%$ & $00.0 \%$ & $415.4 \%$ & 0.274 & 0.138 \\
\hline \multicolumn{2}{|l|}{ Leukotrichia } & $\mathrm{N} \%$ & $416.7 \%$ & $210 \%$ & $00 \%$ & $27.7 \%$ & 0.702 & 0.409 \\
\hline \multicolumn{2}{|l|}{ VASI } & Mean \pm SD & $3.4 \pm 0.9$ & $1.7 \pm 0.4$ & $0.8 \pm 0.2$ & $1.4 \pm 0.4$ & $<0.001$ & $<0.001$ \\
\hline
\end{tabular}

P1, comparison between GG, GA, AA; p2, comparison between GA+AA versus GG; SD, standard deviation.

4. Discussion 
Vitiligo is a chronic systemic acquired disease that has an unpredictable clinical course, characterized by the appearance of macules and achromic or hypochromic patches on the skin and mucous membranes due to the disappearance of melanocytes in the affected area. The cause of vitiligo is not fully understood. There are multiple hypotheses for the pathogenesis of vitiligo which include the genetic, neural, autoimmune, biochemical, and melanocytorrhagy theories [9].

Human $\beta$-defensin (hBD)-1 is an antimicrobial peptide existing in multiple forms ranging from 36-47 amino acids and is encoded by the gene, DEFB1. Classically, hBD-1 is known as the constitutively produced $\beta$-defensin in epithelial cells, produced mainly by epithelial cells in the kidney, lung, female reproductive tract, other mucosal organs and skin. It is thought to have a protective effect against invading organisms, aiding to provide a first-line innate immune defense against invaders [10] , Several gene polymorphisms affecting the HBD gene were studied in relation to different inflammatory and autoimmune diseases [11-13], however, there is a scarcity in the studies discussing the HBD gene polymorphisms' role in vitiligo.

The polymorphism included in the present work was the HBD (-20G/A) gene polymorphism. The wild allele in this SNP is the G allele while the polymorphic allele is the A allele. The A allele decreases the levels of HBD-1 and its gene expression at the tissue level [13].

The current study revealed that HBD (-20G/A) AA genotype and $\mathrm{A}$ allele showed significantly lower frequency in vitiligo patients when compared to the control subjects. Logistic regression analysis revealed that (-20G/A) AA genotype and A allele exerted a protective effect against vitiligo development. Similar protective effect was reported against other autoimmune diseases [11-13].

Concerning VASI score, it decreased gradually between -20G/A GG, GA, AA respectively. With prediction of higher VASI score in vitiligo cases, using age, gender, smoking, FH, BMI, onset, course, duration, DEFB1 genotypes as covariates, -20G/A (GA+AA) was associated with prediction of lower VASI score. Moreover, carriers of AA genotype of HBD1 (-20G/A) SNP had significantly lower VASI score.

The HBD possess a significant role in the pathogenesis of autoimmunity via its chemoattractant properties for immature dendritic cells and memory $\mathrm{T}$ cells, which in the presence of 'danger' signals (e.g. oxidative stress, high levels of IL-6, IL-8 and heatshock protein 70) would promote initial autoantigen presentation [14] and depigmentation flares (Yang et al., 2007). HBD1 (-20G/A) A allele protective effect can be explained by the hypothesis that HBD1 (-20G/A)A allele appears to be related to lower both HBD-1 and gene expression at the tissue level.[13] This could result in lower inflammatory and autoimmune burden to the melanocytes.

The studied gene polymorphism in the SNPs HBD1 (-20G/A) is related to the development of vitiligo in an Egyptian sample with protective effect.

\section{Conclusion}

From this study we can conclude that HBD (20G/A) AA genotype and A allele exerted a protective effect against vitiligo.

\section{Financial support and sponsorship Nil.}

\section{Conflicts of interest}

There are no conflicts of interest

\section{Reference}

[1] Y.Zhang, Y.Cai, M.Shi, The prevalence of vitiligo: a meta-analysis. PloS one. 27, Vol.11(9), PP.e0163806, 2016.

[2] C.W. Choi, S.H. Eun, K.H.Choi, Increased risk of comorbid rheumatic disorder in vitiligo patients: a nationwide population-based study. J Dermatol , Vol.44, PP. 909-13, 2017.

[3] Y. Zhen, L. Yao, S .Zhong, Y. Song, Enhanced Th1 and Th17 responses in peripheral blood in active non-segmental vitiligo. Archives of dermatological research, Vol.308(10), PP.703-10, 2016.

[4] D. Yang, Z.H. Liu, P .Tewary, Q.Chen, Defensin participation in innate and adaptive immunity. Current pharmaceutical design, Vol.13(30), PP.3131-9, 2007.

[5] S. Vordenbäumen, M. Schneider, Defensins: potential effectors in autoimmune rheumatic disorders. Polymers, Vol.3(3), PP.1268-81, 2011.

[6] V.Polesello, L.Zupin, R.Di Lenarda, M.Biasotto, DEFB1 polymorphisms and salivary hBD-1 concentration in oral lichen planus patients and healthy subjects. Archives of Oral Biology, Vol.73, PP.161-5, 2017.

[7] E.Ozlu, A.S.Karadag, S.Ozkanli, S.Oguztuzun, The investigation of antimicrobial peptides expression and its related interaction with methotrexate treatment in patients with psoriasis vulgaris. Cutaneous and ocular toxicology, Vol.36(4), PP.321-6, 2017.

[8] H.Hamzavi, D.Jain, J.McLean, H.Shapiro, Parametric modeling of narrowband UV-B phototherapy for vitiligo, using a novel quantitative tool: the Vitiligo Area Scoring Index," Archives of Dermatology, vol. 140(6), PP.677-683, 2004.

[9] M.L.Frisoli, K.Essien, J.E.Harris, Vitiligo: Mechanisms of Pathogenesis and Treatment. Annual Review of Immunology, Vol.38, PP.62148, 2020. 
[10] V. Ryan, A.K.Bhunia, Mitigation of foodborne illnesses by probiotics. InFoodborne Pathogens,PP. 603-634. Springer, Cham, 2017.

[11] P.Sandrin-Garcia, L.A.Brandão, R.L.Guimarães, J.A.Pancoto, Functional single-nucleotide polymorphisms in the DEFB1 gene are associated with systemic lupus erythematosus in Southern Brazilians. Lupus, Vol.21(6), PP.625-31, 2012.

[12] B.C.Németh, T.Várkonyi, F.Somogyvári, C.Lengyel, Relevance of $\alpha$-defensins (HNP1-3) and defensin $\beta-1$ in diabetes. World
J.,gastroenterology: WJG, Vol.20 (27), PP. $.9128,2014$.

[13] V.Zanin, L.Segat, A.M.Bianco, L.Padovan, DEFB1 gene 5'untranslated region (UTR) polymorphisms in inflammatory bowel diseases. Clinics, Vol.67(4), PP.395-8, 2012.

[14] P.Manga, N.Elbuluk, S.J.Orlow,Recent advances in understanding vitiligo. F1000Res, Vol.5, PP.2234, 2016. 